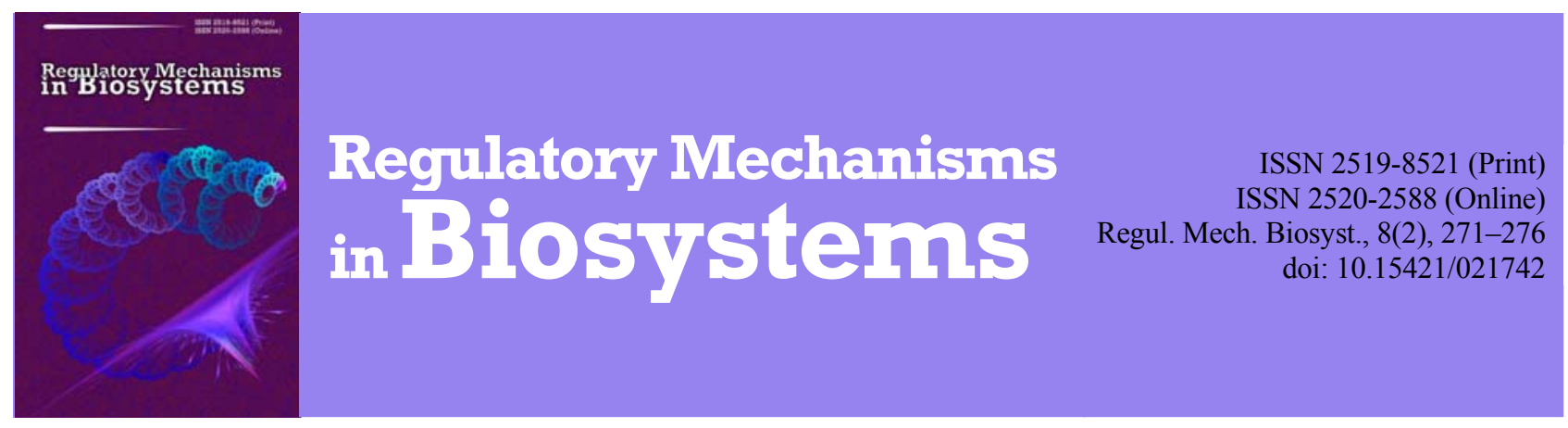

\title{
Immunohistochemical and clinical changes in the respiratory tract of chickens, naturally infected with the fowlpox virus
}

\author{
M. S. M. Al Rawashdeh \\ Mutah University, Al-Karak, Jordan
}

Article info

Received 24.03.2017

Received in revised form 20.04.2017

Accepted 23.04.2017

Animal Production Department School of Agriculture, Mutah University, Al-Karak, P.O. Box 7, 61710, Jordan.

Tel: 00962-799-973-354.

E-mail:Drmsmr77@yahoo.com

\begin{abstract}
Al Rawashdeh, M. S. M. (2017). Immunohistochemical and clinical changes in the respiratory tract of chickens, naturally infected with the fowlpox virus. Regulatory Mechanisms in Biosystems, 8(2), 271-276. doi:10.15421/021742
\end{abstract}

It is clear that currently the question of study of the pathological changes in the respiratory tract of chickens due to the impact of the fowlpox virus remains relevant, as the pathogenesis of nutritional deficiency, the presence of mycotoxins or the papilloma virus are characterized by the same clinical manifestations. We analyzed 88 chickens, naturally infected with the fowlpox virus, using clinical and immunohistochemical methods of investigation. Among all species of birds, we studied only chickens, data on which were collected for five years from veterinary clinics. The investigation revealed lesions basically characterized by the presence of changes in the typical structure of the chicken's respiratory tract. As a result, we found clear criteria for the pathological process in the respiratory tract of chickens, which are typical for fowlpox. Thus, we discovered respiratory tract obstruction, as well as many intracytoplasmic pale eosinophilic inclusions in hyperplastic cells. We found an accumulation of mononuclear cells consisting mainly of macrophages, lymphocytes, plasma and mononuclear cells inside the mucous and muscle membranes. Bronchial lumens were blocked by necrotic and desquamated epithelial cells, red blood cells, bacterial colonies and amorphous eosinophilic material. We found accumulations of lymphocytes and macrophages in the parenchyma of the lungs. The hyperplastic epithelial cells reacted immunohistochemically with antibodies against the fowlpox virus in the respiratory tract. Immunoreaction occurred mainly in the cytoplasm of infected cells, inclusions, and necrotic and desquamated cells. The study proved immunohistochemical methods of investigation can be a useful additional tool for establishing a final diagnosis, especially in acute and subacute phases of the disease. The following respiratory signs were observed in severe cases of fowlpox: damage to the lungs in 33 cases (46.5\%), parabronchium $20(28.2 \%)$, parabronchial connective tissue $-8(11.3 \%)$, and mucous membrane of the larynx and trachea -10 $(14.1 \%)$. In mild and moderate cases of fowlpox, the following respiratory signs were observed: hyperemia and thickening of the mucous membrane of the trachea - $14(82.4 \%)$, as well as hyperemia in the nasal conchae and paranasal sinuses $-3(17.7 \%)$. In the future, it will be necessary to conduct deeper studies to detect pathological manifestations of this disease, not only in the respiratory tract, but also in other organs and systems of chickens.

Keywords: study of pathogenesis; immunological disorders; histological changes; viral infection

\section{Introduction}

According to the literature, fowlpox is a disease that is widespread all over the world and is caused by the virus of the family Poxviridae, the genus Avipoxvirus (Fauquet, 2005; Tripathy, 2000). In addition, it is known that in the bird population its incidence is about $10 \%$ to $95 \%$, and the mortality rate is about $50 \%$. In fact, chickens are the main hosts of the fowlpox virus, but other bird species such as pheasants, peacocks, and turkeys (Medina et al., 2004) can be affected as well. Moreover, fowlpox is characterized by large economic losses, which is mainly due to the decline in egg production and excess mortality among birds (Ali et al., 2016; Bande et al., 2016).

There is a wealth of data in the literature various forms of the course of the disease. Thus, it has been reported that fowlpox is a slowly spreading viral infection which is often characterized not only by proliferative skin lesions, but also by changes in the upper gastrointestinal tract, respiratory tract (diphtheria form) (Singh et al., 1987; Docherty et al., 1991; Fallavena et al., 1993; Gortázar et al., 2002; Cavanagh, 2007; Bande et al., 2016).

However, it should be noted that this disease can occur in any age group of birds, but young birds are considered to be the most vulnerable age group (Smits et al., 2005). There are also no specific data on the time of year in which fowlpox is most prevalent, however, there are some data which indicate that the disease most often poses a risk to chickens during the warm months of the year. Along with that, the virus can be present in abundance in the focal points of infection and transmitted by contact through skin abrasions. Skin lesions (crusts), which are separated as a result of removal of birds from poultry houses, can also become the source of aerosol infection. Mosquitoes and other biting insects can serve as mechanical vectors of the disease. In addition, the disease tends to persist for extended periods in a birds of different ages in poultry production units due to the slow spread of the virus and the presence of susceptible birds (Smits et al., 2005).

So, the authors call attention to the fact that the fowlpox virus is considered sustainable in the environment over the course of observation. It has been reported that there are most likely special genes in the genome of the fowlpox virus that protect the virus from environmental hazards. In this case, the fowlpox virus is destroyed only at extremely high temperatures. Drying and cold can preserve it. In lyophilized form (a soft drying method, in which the freezing is first carried out, followed by putting a material into a vacuum chamber for sublimation of the solvent), it persists for up to 8 years, 
and in pathological material (crust) at $-15^{\circ} \mathrm{C}$ remains virulent for more than two years (Smits et al., 2005).

According to the literature, the virions of the fowlpox virus have an oval or rectangular shape, sizes about $140-450 \mathrm{~nm}$, as well as a complex type of symmetry. The virions of the fowlpox virus consist of a nucleoid containing a genome, lateral bodies and an external lipoprotein shell forming protrusions (da Silva et al., 2009).

Since it is known that viruses of different genera differ in biological properties, the principal properties are as follows: spectra of pathogenicity, virulence and hemagglutinating activity, according to which they differ significantly among themselves. In addition, all fowlpox viruses are DNA genomic and have a virion containing enzyme for their own transcription. In this connection, all viruses of this family replicate in the cytoplasm of cells without the need for cellular transcription enzymes. It is also important to note that strains of the fowlpox virus are identical in antigenic terms. It has been noted that field and vaccinal strains of the disease have only minor differences in the genomic profile (Smits et al., 2005).

In addition, structurally the virions of the fowlpox virus contain an external protein antigen as part of the surface villi (filaments), which can induce the formation of antihemagglutinating virus neutralizing antibodies, which in turn ensures the immune development after the disease. Internal polypeptides induce complementfixing and precipitating antibodies (da Silva et al., 2009). Interestingly, the antigenic variability in this type of virus has not been established. However, strains of the fowlpox virus differ not only in the pathogenicity spectrum, but also in virulence (da Silva et al., 2009).

It is shown that the fowlpox virus is able to agglutinate erythrocytes of chickens of some species $(0.5 \%$ suspension of erythrocytes in physiological saline, $\mathrm{pH} 7.2-7.4,37^{\circ} \mathrm{C}$ ). When preserved with $0.5 \%$ formalin and stored at $4^{\circ} \mathrm{C}$, the suspension of the virus retains hemagglutinating activity for more than 8 months and can be used in the hemagglutination and hemagglutination inhibition reactions (da Silva et al., 2009).

It is emphasized that the experimental infection is reproduced on nonimmune chicks and chickens when infected in a scarified crest, jowls and feather follicles (da Silva et al., 2009).

The incubation period of the disease lasts an average of 15 to 20 days. Further, sick birds show deteriorating appetite, lethargy, decline in egg production. The course of the disease can acquire cutaneous, diphtheritic or mixed forms. In diphtheritic form of fowlpox, lesions develop on the mucous membranes of the mouth, esophagus, pharynx, larynx and trachea. Sometimes lesions are observed in one or more of these organs (Pringle, 1999). In addition, scaly patches, closely adjacent to the mucous membrane of the larynx and oral cavity, or proliferative masses may develop. However, damage to the oral cavity often interferes with the birds' ability to feed. Tracheal lesions affect the respiratory tract. Mortality is usually high in the case of diphtheric infection, and can reach 70\% (Bande et al., 2016).

Pathologicoanatomic changes are quite specific and are based primarily on the form of the course of the disease. Most often, ochroleucous soaring spots of different size, sometimes covered with red bloodstains, caseous masses or pseudomembranes in the respiratory tract and intestines (da Silva et al., 2009) can often be found in the patterns of dead birds. In the body of a sick bird, the virus is localized in the pock marks of the featherless areas of the head, on the pharyngeal, laryngeal, and tracheal mucosa. On the 17-20th day after infection, the virus can be found in the blood, kidneys, ovaries, spleen, brain. A bird which has experienced the disease retains a long-standing virus infection carrier state (for more than 10 months) (da Silva et al., 2009).

The literature actively discusses the issue of choosing a method for diagnosing fowlpox, which is usually based on epizootological, clinical, pathologicoanatomic and laboratory data. However, some scientists note that the diagnosis should be based primarily on laboratory tests (da Silva et al., 2009). Thus, histological examination of cutaneous or diphtheritic lesions reveals epithelial hyperplasia with intracytoplasmic inclusions in the affected cells. Elementary bodies can be found in smears of lesional tissues using the Gimenez staining technique. The researchers differentiate the diphtheritic form of fowlpox with tracheal injury from infectious laryngotracheitis, which is caused by the herpes virus and characterized by the presence of nucleoids (Ali et al., 2016; Bande et al., 2016).

Microscopic examination of invaded tissues stained with hematoxylin and eosin reveals eosinophilic cytoplasmic inclusions, which are also detected with fluorescent antibodies and immunohistochemical methods (Ali et al., 2016; Bande et al., 2016). Viral particles with a typical virus morphology are determined by transmission electron microscopy with negative coloration, as well as in ultrathin sections of lesions (Preis et al., 2014).

Immune responses to the fowlpox virus can be detected by virus neutralization, agar gel immunodiffusion, immunofluorescence, passive hemagglutination tests, enzyme immunoassay and immunoblotting (Ali et al., 2016; Bande et al., 2016). There are also indications in the literature that there is no cure for fowlpox. That is why chickens at poultry farms should be vaccinated with a virus vaccine derived from live embryos or a cell culture. The most widely used vaccines are attenuated fowlpox viruses and varicella-zoster viruses with high immunogenicity and low pathogenicity. In high-risk areas, vaccination with an attenuated vaccine in the first few weeks of life and revaccination between 12 and 16 weeks is often a sufficient preventive measure against the onset of the disease. The naturally infected or vaccinated birds develop a humoral, as well as a cellmediated response (Shi et al., 2011).

Jordan has developed and currently uses two types of live respiratory vaccines: from a non-reactogenic heterologous strain of the fowlpox virus of New Jersey and from a naturally attenuated fowlpox cholera strain of AS-27, as well as a vaccine from an attenuated cultural strain of the fowlpox virus that is injected into the wing web. In vaccinated chicks immunity is maintained for an average of 3 to 6 months, in chickens up to 10 months.

Thus, fowlpox can be diagnosed by a veterinarian based on the history of the flock, the presence of typical lesions, and in some cases by microscopic and immunohistochemical examination of affected tissues (Ali et al., 2016; Bande et al., 2016).

However, it should be noted that currently there are reports in the literature that it is the immunohistochemical method of studying the fowlpox virus that is the most common and quickest way to diagnose the disease (Crespo et al., 2007).

In view of the above, the focus of this paper was to study immunohistochemical and clinical changes in the respiratory tract of chickens, naturally infected with the fowlpox virus.

\section{Materials and Methods}

Among all species of birds we dealt with chickens only, data on which had been collected from veterinary clinics for 5 years. The study included 88 chickens naturally infected with the fowlpox virus examined using clinical and immunohistochemical methods. Paranasal sinuses, nasal conchae, larynx, trachea, bronchi, parabronchial connective tissue, air sacs, and lungs served as samples for histopathological examination (Tripathy et al., 2000). The studied chickens weighed from $40.0 \mathrm{~g}$ to $3.5 \mathrm{~kg}$. Pockmarks (crusts) were cut out of the sick birds and placed in a $50 \%$ glycerin solution and delivered to the laboratory in a thermos with a cooling mixture. The pockmarks were used directly to make impression smears, with subsequent drying, fixing in an alcohol ester mixture, staining and examination. The preserved pockmarks (crusts) were used to prepare a $10 \%$ suspension, which was used to isolate the virus.

Laboratory diagnostics included the identification of fowlpox virions (elementary bodies) by transmission electron microscopy virusoscopy, chick-embryo culture, as well as by immunofluorescence test (IFT) and diffusion precipitation test in the gel (DPT).

Impression smears were stained by Morozov silver impregnation. The smears were covered with Ruge's liquid (reagent No. 1: $1.0 \mathrm{ml}$ of glacial acetic acid, $2.0 \mathrm{ml}$ of a $40 \%$ formaldehyde solution and $100 \mathrm{ml}$ of distilled water) for 1 minute; washed with distilled water; etched with tannin solution (reagent No. 2: $1 \mathrm{~g}$ of 
tannin and $5 \mathrm{ml}$ of distilled water) for 1 to 2 minutes, preheated until the appearance of fumes; washed and treated with a solution of ammonia silver (reagent No. 3: $5 \mathrm{~g}$ of ammoniacal silver crystals were dissolved in $100 \mathrm{ml}$ of distilled water, and a $25 \%$ ammonia solution was added dropwise to $80 \mathrm{ml}$ of the resulting solution until the resolution of precipitate; for staining the resulting reagent was diluted with distilled water 1:10); slightly warmed to a dark brown colour of the smear; washed with distilled water and viewed under immersion oil using a transmission electron microscope. Virusoscopy was considered positive only in the presence of characteristic dark brown formations (Borrel elementary bodies - fowlpox virions) located in abundance throughout the yellowish smear.

Retrospective diagnosis of the fowlpox virus is rare. In the case of an expired course of the disease, chickens without visible clinical signs have paired sera examined by diffuse precipitation reaction (DPR). However, the precipitating serum of convalescents often appears to be subactive, with no antibodies detected in $15 \%$ of chickens with fowlpox. That's why we did not resort to this kind of diagnostic.

DPR strengths: simplicity of technique; quick response time; possibility of photographic recording of the result.

A bioassay test at negative virusoscopy was performed on 10 to 12-day chicken embryos, with the fowlpox virus isolated from pathological material. Chicken embryos were infected on the chorion allantoic and amniotic sacs (CAS), and incubated for 5 to 6 days. The detection of specific inclusion bodies (Bollinger bodies) in the cytoplasm of infected cells was an indicative method of indicating the fowlpox virus. Individual or homogenous pockmarks on CAS as well as a significant thickening or a tremelloid edema of the whole CAS (with a high infectious dose) were considered an indication of the virus replication. In the absence of pockmarks, three blind passages were made. CAS pockmarks were used to make impression smears and virusoscopy.

Sometimes, scientists also recommend that a bioassay test be performed on 3-4-month-old chicks which are nonimmune to the fowlpox virus and are inoculated with a $10 \%$ suspension of the pathological material by rubbing it (epicutaneously) into the scarified surface of the crest, or into the feather follicles of the drum. If there is a virus in the material, the variolar tissue lesions develop in the place of rubbing on the 5-6th day. The specific character of these lesions is identified by smear virusoscopy.

For stained sections established practices were used. For general examination, sections were stained with hematoxylin-eosine, with connective tissue stained to van Gieson. Histochemical methods involved Sudan III total grease staining, Brachet nucleic acid staining, mucicarmine staining. Hemosiderin pigment was identified by Perls staining, with glycogen and alkaline phosphatase activity identified by Iabadai and Gomori staining, respectively (Tripathy et al., 2000). Specimens were cut into 4 micron thick pieces, which were evaluated using a transmission electron microscope following dehydration (Tripathy et al., 2000).

To study immunohistochemical changes in tissues, material samples were stained with immunoperoxidase using polyclonal hyperimmune serum. All sections were dewaxed in xylene and rehydrated by means of alcohols. Endogenous peroxidase was blocked by means of 3\% hydrogen peroxide. After washing with phosphate buffer for 5 minutes, specimen slides were incubated with normal rabbit serum for 30 minutes at an ambient temperature. Sections were incubated with polyclonal hen's hyperimmune serum against the fowlpox virus with $1 / 64$ concentration in phosphate buffer for 30 minutes. Tissues were rinsed in phosphate buffer after each stage. Primary antibodies were omitted and replaced with a phosphate control buffer (Tripathy et al., 2000).

The arithmetic mean of the initial data and the Student's t-test - a method for estimating the significance of differences in mean values were used as the basic statistical parameters. The Student's t-test allowed us to check the difference of mean values in two samples.

The statistical significance of the findings was a measure of reliability ("representative sampling"). The statistical data processing used methodological instructions on the principal methodological methods of statistical analysis in biological studies.

\section{Results}

In describing the abnormalities in occurring in chickens with this disease, we used the clinical features characterized by the presence of apathy in birds, as well as dyspnoea from moderate to severe severity. Some hens revealed conjunctival redness, palpebral and paranasal sinus edema. Studies have shown that tissue lesions were also characterized by typical changes in the structure of the respiratory tract of chickens. It was found, that in 71 individuals $(80.7 \%)$ the disease was considerably severe, with 17 (19.3\%) cases demonstrating mild to moderate severity.

When studying the course of the disease in birds with a severe degree of course of the disease, the following respiratory signs were observed: pulmonary involvement in 33 cases (or 46.5\%), parabronchi in 20 cases $(28.2 \%)$, parabronchial connective tissue in 8 cases $(11.3 \%)$, as well as laryngeal and tracheal mucosa in 10 cases $(14.1 \%)$. When studying the course of the disease in chickens in cases of mild and moderate severity, the following respiratory signs were observed: tracheal blennosis in 14 cases $(82.4 \%)$, as well as hyperemia in the nasal conchae and paranasal sinuses in 3 cases $(17.7 \%)$.

The course of fowlpox was characterized by the appearance of skin lesions on various parts of the body and mucous membranes of the nasal and oral cavities. The abnormalities in fowlpox were notable for their staging: macular erythema (local redness of the skin area), papule (compaction of the skin area due to cellular infiltration), vesicle (filling with vesicular serous fluid), pustule (transition of serous inflammation to purulent) and crust (appearance of crusts at the site of tissue lesion).

Fowlpox virus was confirmed in the pathological material, with characteristically scattered virions found in the smears of the affected areas. Histological examination of the material from the affected areas was carried out simultaneously with the detection of elementary bodies. At the same time, cytoplasmic inclusions, intranuclear vacuoles, epidermal hyperplasia of the skin were detected. A positive result of viroscopic examination afforded grounds for final diagnosis of fowlpox. Thus, respiratory obstruction and numerous pale eosinophilic intracytoplasmic inclusions were identified in the hyperplastic cells. The interior of lamina propria of mucosa and muscular tunic of pharynx revealed accumulation of mononuclear cells consisting mainly of macrophages, lymphocytes, plasma and mononuclear cells.

Bronchial lamina were covered by necrotic and desquamated epithelial cells, red blood cells, bacteria colonies and amorphous eosinophilic material. Accumulations of parenchyma cells and macrophages were found in the pulmonary parenchyma. Thus, histological examination of lesions in all chicken specimens showed similar pathology. Epithelial cells were swollen, roundshaped, separated from one another. The cytoplasm of hyperplastic epithelial cells contained typically substantial eosinophilic inclusions (identified as Bollinger bodies). Inclusions stretched cytoplasm of cells, resulting in their death.

Virusoscopy was considered positive, as we detected characteristic dark brown formations (Borrel elementary bodies fowlpox virions) located in abundance throughout the yellowish smear. Viruses were large, comparable to the size of the smallest bacteria. Viruses were notable for a complex type of symmetry due to the presence of two additional structures that make up the virion. They represented the membranes, with the outer one having a greater thickness and a large number of hollow helicoidal filaments without definite ordering constituting the lipoprotein shell of the virions, which is a characteristic morphological sign of this virus. The nucleoid at the heart of the virion had a characteristic biconcave shape. The mature virion had a size of about $330 \times 280 \times$ $200 \mathrm{~nm}$. The surface of the virions was covered with tubular structures with an average size of $8 \mathrm{~nm}$. So, we found that the 
morphology of the fowlpox virus was similar to the morphology of other viruses of the family Poxviridae. It has been proven that the inclusions of fowlpox consisted of lipids.

Our study showed that a notable increase in the number of lipidic vacuoles can be instrumental in the formation of inclusion bodies, since we observed an initial localization of the virus around the lipidic vacuoles. Further, the staining confirmed that there were empty vacuoles at the heart of the inclusion bodies. Thus, it can be concluded that the center of inclusions can contain lipidic vacuoles and lipid granules, which can be instrumental in the formation of inclusion bodies.

Changes in the material under examination, which were caused by the impact of the fowlpox virus, were mainly characterized by degeneration and proliferation of epithelial cells, as well as the formation of cytoplasmic inclusions. Hyperplastic lesions caused by the fowlpox virus were classified by abnormalities in tissues close to tumoral and inflammatory processes. We observed that the hyperplastic epithelial cells containing cytoplasmic inclusions were separated from one another. In addition, it was found that the inclusions increased in size and stretched epithelial cells, resulting in degeneration and cell death. Thus, it can be assumed that the epithelial cell changes found in our study might have been caused by viral replication in the cells.

Our study revealed that it was the epithelium of the lower respiratory tract that was significantly affected in the chickens, which was characterized not only by hyperplasia of epithelial cells in the trachea and bronchi, but also by accumulation of mononuclear cells in the underlying layers, tissue damage near the bronchi, which confirms the severe degree of course of this infection. The obtained data presumably bear witness to the multiplication of the fowlpox virus in the lungs through the lymphatic or circulatory system.

In this study, the immunohistochemical diagnosis was confirmed in $88(100 \%)$ cases, which indicates the high performance of this method for diagnosing viral diseases in birds. Immunohistochemical study of material collected from the chickens' respiratory tracts revealed that the hyperplastic epithelial cells reacted with antibodies against the fowlpox virus. Immunoreaction occurred mainly in the cytoplasm of infected cells, in inclusions, desquamated and necrotic cells. In addition, the presence of matrix inclusions was determined using immunoperoxidase. This study showed that immunoperoxidase based diagnostics can be used in the establishing of a morphological diagnosis and detection of infection of the fowlpox virus at early stages of the disease.

The obtained results indicate the need for further research in this field, especially for the detection of pathological and immunohistochemical manifestations of this disease not only in the respiratory tract, but also in other organs and systems of chickens. Despite the fact that a multilevel study is cost-intensive and potentially time consuming, it is precisely special and comparative methods of studying viral particles using immunoperoxidase which can be used to conduct immunoreactions as part of experimental studies. Thus, this study has shown that immunohistochemical methods of research can be successfully applied in multilevel studies, which is especially important in the case of loss of egg production by birds at poultry farms.

\section{Discussion}

Despite the fact that the incidence of mortality due to fowlpox has been significantly reduced of late, this disease remains damaging for producers of meat and eggs of birds, as evidenced by data from the literature (Ali et al., 2016; Bande et al., 2016). According to researchers, members of the genus Avipoxvirus typically infect birds (Vanderplasschen, and Pastoret, 2003).

It has been reported that Avipoxvirus is caused by symptoms such as the appearance of pustules in the skin of birds, as well as signs of disease similar to diphtheria, which may include characteristic necrotic lesions covering the oral cavity and upper respiratory tract (Zhao et al., 2014), which may be due to the fact that, like other avian viruses, the fowlpox virus can be transmitted through vectors mechanically, for example, through mosquitoes. Other authors note that in this disease the virus affects the mucous membranes of the pharynx, larynx and, more rarely, the epithelium of the lower respiratory tract (Bande et al., 2016).

According to the findings of other scientists, lesions of the skin and respiratory tract are often found only in domesticated and wild birds infected with Avipoxvirus (da Silva et al., 2009). However, there are two common forms of clinical signs. The cutaneous form is considered the most common and manifests itself in the form of featherless carbuncles around the eyes and other areas of the skin of chickens, sometimes in large clusters. The diphtheritic variety of fowlpox is characterized by the presence of elevated yellowish spots on the mucous membranes of the mouth, esophagus, trachea and lungs, which can result in dyspnoea or swallowing. In both cases, birds may be weak and exhausted (Zhao et al., 2014). We have also found that the clinical signs of the disease in chickens were characterized by lethargy, mild to severe dyspnoea, conjunctival redness, excessive tearing, palpebral and paranasal sinus edema.

In addition, our studies have shown that tissue lesions are mainly characterized by typical changes in the structure of the respiratory tract of chickens. The obtained data coincide with the data of other scientists (Beytutl, and Haligür, 2007), who noted similar manifestations in chickens during a clinical research.

We have found that the following respiratory signs were observed in birds suffering from mild to moderately severe cases of the disease: pulmonary involvement in $46.5 \%$ ), parabronchi in $28.2 \%$, parabronchial connective tissue in $11.3 \%$, as well as laryngeal and tracheal mucosa in $14.1 \%$. In the course of disease, the following was observed in birds with mild to moderate respiratory signs: hyperemia and thickening of tracheal mucosa in $82.4 \%$, as well as hyperemia in the nasal turbinates and paranasal sinuses in $17.7 \%$. The literature review also showed that in most birds the disease typically occurs with mild severity and rarely leads to death, particularly in the cutaneous form of the disease (da Silva et al., 2009). However, our study showed that in $80.7 \%$ the disease was of considerable severity, with only $19.3 \%$ demonstrating an average degree of severity of the disease process. It should be noted that we studied a separate form of the disease that affects just the respiratory system of chickens.

In addition, our study showed a heavy mortality among chickens, naturally infected with fowlpox virus. The literature has such data in this regard. Thus, the mortality rate is usually high when the fowlpox virus affects the mucous membrane of the oral cavity and upper respiratory tract, or when a flock is affected by a consecutive infection or maintained in not sufficiently sound environmental conditions (Singh et al., 1987; Docherty et al., 1991; Fallavena et al., 1993; Gortázar et al., 2002; Cavanagh, 2007).

We hypothesized that the high mortality rate of birds found in this study could be caused by consecutive infection, decreased chick immunity or unusual pathogenicity of the pathogen, as the birds were kept in satisfactory environmental conditions.

There is evidence that changes due to the fowlpox virus are mainly characterized by degeneration and proliferation of epithelial cells, as well as formation of cytoplasmic inclusions (Bande et al., 2016), as observed in this study. In addition, the resulting hyperplastic lesions caused by the fowlpox virus were classified by abnormalities in tissues close to tumoral and inflammatory processes.

It is known that Avipoxvirus is a brick-shaped virus typically 200 $\mathrm{nm}$ in diameter, which is much larger than regular viruses, whose diameter is about $60 \mathrm{~nm}$ (da Silva et al., 2009). Our study showed that the morphology of the fowlpox virus was similar to that of other viruses of the Poxviridae family, with the elementary body having brick shape and size of about $330 \times 280 \times 200 \mathrm{~nm}$, which concurred with the data from the literature (da Silva et al., 2009).

The factual findings furnish convincing proof that viral replication occurs in the cytoplasm of virus-infected epithelial cells, which has also been noted by various scientists (da Silva et al., 2009). 
According to the findings of this research, the virion consisted of a dense centered biconcave nucleus or nucleoid with two lateral bodies in each concave and surrounded by a shell, as noted by many authors (Tripathy, 2000). In this study, it was noted that the inclusions consisted of lipids. Previous researchers also suggested that the inclusion of fowlpox viral particles might have an outer layer that can be lipid by nature of origin (da Silva et al., 2009). Our study also showed that a notable increase in the number of lipidic vacuoles can be instrumental in the formation of inclusion bodies, since we observed an initial localization of the virus around the lipidic vacuoles. In addition, staining showed that there were empty vacuoles at the heart of the inclusion bodies. Thus, it can be concluded that the center of inclusions can contain lipidic vacuoles and lipid granules, which can be instrumental in the formation of inclusion bodies. These findings were similar to previous studies (Halıür et al., 2009).

In previous studies, viral particles were described at various stages of development during electron microscopy examination (da Silva et al., 2009). In our study, intracytoplasmic inclusions were presented, which resembled classical inclusions easily detectable with a light microscope. We observed that the hyperplastic epithelial cells containing cytoplasmic inclusions were separated from one another (Tanizaki et al., 1989). In addition, it was found that the inclusions increased in size and stretched epithelial cells, resulting in degeneration and cell death. Thus, it can be argued that the epithelial cell changes found in our study could be caused by viral multiplication in cells, which concurs with the factual findings (Reed, and Schrader, 1989; Tanizaki et al., 1989; Pringle, 1999; Smits et al., 2005; Shi et al., 2011; Susta et al., 2013; Preis et al., 2014; Ali et al., 2016).

There is evidence in the literature that reproduction of the fowlpox virus in the lungs is possible through the lymphatic or circulatory system (Singh et al., 1987). In our study, the lower respiratory epithelium was significantly affected in chickens, which was characterized by hyperplasia of epithelial cells in the trachea and bronchi, accumulation of mononuclear cells in the underlying layers, tissues lesions close to bronchi, thus proving a persistent infection.

In this study, the immunohistochemical diagnosis was confirmed in $100 \%$ cases, which indicates the high performance of this method for diagnosing viral diseases in birds. In addition, the presence of matrix inclusions was determined using immunoperoxidase. Thus, this study showed that immunoperoxidase based diagnostics can be used in the establishing of a morphological diagnosis and detection of infection of the fowlpox virus at early stages of the disease.

We concluded that although a multilevel study is cost-intensive and potentially time consuming, it is precisely special and comparative studies of viral particles using immunoperoxidase which can be used to conduct immunoreaction in experimental studies, which concurs with research data of other scientists (Haligür et al. 2009). Thus, this study has shown that immunohistochemical research methods can be successfully applied in multilevel studies, which is especially important in case of loss of egg production in birds at poultry farms, since our study confirmed the very high sensitivity of these techniques at the early stage of diagnosing fowlpox.

\section{Conclusion}

It has been established that up to the present the issue of study of pathological changes in the respiratory tract of hens due to exposure to the fowlpox virus continues to be relevant since pathogenesis of deficiency of nutrients, presence of mycotoxins or papilloma virus is characterized by identical clinical implications. We hypothesized that the high mortality rate of chickens found in this study could be caused by consecutive infection, decreased bird immunity or unusual pathogenicity of the pathogen, as the chickens were kept in satisfactory environmental conditions. It was revealed that the tissue lesions in chickens were mainly characterized by the presence of changes in the typical structure of the respiratory tract and skin. Clear criteria were clarified for the pathological process in the respiratory tract of chickens, which are specific for this type of fowlpox. It was found that the morphology of the fowlpox virus was similar to that of other viruses of the family Poxviridae. It is assumed that the epithelial cell changes found in our study might have been caused by viral replication in the cells. It was established that it is the epithelium of the lower respiratory tract that was significantly affected in the chickens, which was characterized not only by hyperplasia of epithelial cells in the trachea and bronchi, but also by accumulation of mononuclear cells in the underlying layers, tissue damage near the bronchi, which confirms the severe degree of course of this infection. The obtained data presumably bear witness to the multiplication of the fowlpox virus in the lungs through the lymphatic or circulatory system. It is proved that a noticeable increase in the number of lipidic vacuoles can be instrumental in the formation of inclusion bodies.

Immunohistochemical diagnosis was confirmed in $100 \%$ of cases, which indicates the high performance of this method for diagnosing viral diseases in birds, which can also be used in the future as the most common and quickest way of diagnosing the fowlpox virus. It has been shown that immunohistochemical methods of research can be successfully applied in multilevel studies, which is especially important in the case of loss of egg production by birds at poultry farms. In the future, more in-depth studies should be conducted to identify pathological and immunohistochemical manifestations of this disease not only in the respiratory tract, but also in other organs and systems of chickens.

\section{References}

Ali, A. M. A., Mansour, S. M., Fathy, A. M., \& Eid, A. A. (2014). Detection of avian poxvirus in an egyptian goose (Alopochen aegyptiacus). Global Animal Science Journal, 1(3), $64-68$.

Bande, F., Arshad, S. S., Omar, A. R., Bejo, M. H., Abubakar, M. S., \& Abba, Y. (2016). Pathogenesis and diagnostic approaches of avian infectious bronchitis. Advances in Virology, 2016, article ID 4621659.

Cavanagh, D. (2007). Coronavirus avian infectious bronchitis virus. Veterinary Research, 38(2), 281-297.

Da Silva, P. S., Batinga, T. D. B., Sales, T. S., Herval, E. F. G., Ramos, I., Maia, P. C. C., \& Fernandes, L. M. B. (2009). Fowlpox: Identification and adoption of prophylactic measures in backyard chickens in Bahia, Brazil. Revista Brasileira de Ciência Avícola, 11(2), 115-119.

Docherty, D. E., Long, R. I., Flickinger, E. L., \& Locke, L. N. (1991). Isolation of poxvirus from debilitating cutaneous lesions on four immature grackles (Quiscalus sp.). Avian Diseases, 35(1), 244-247.

Fallavena, L. C. B., Rodrigues, N. C., Scheufler, W., Martins, N. R. S., Braga, A. C., Salle, C. T. P., \& Moraes, H. L. S. (1993). Atypical fowlpox in broiler chickens in southern Brazil. Veterinary Record, 132(25), 635.

Fauquet, C. M., Mayo, M. A., Maniloff, J., Desselberger, U., \& Ball, L. A. (2005). Virus taxonomy: VIIIth report of the International Committee on Taxonomy of Viruses. Elsevier Academic Press.

Weli, S. C., \& Tryland, M. (2011). Avipoxviruses: Infection biology and their use as vaccine vectors. Virology Journal, 8, 49.

Gortázar, C., Millán, J., Höfle, U., Buenestado, F. J., Villafuerte, R., \& Kaleta, E. F. (2002). Pathology of avian pox in wild red-legged partridges (Alectoris rufa) in Spain. Annals of the New York Academy of Sciences, 969(1), 354-357.

Halıür, M., Özmen, Ö., Vural, S. A., \& Berkin, Ş. (2009). Pathological, immunohistochemical and electron microscopical examinations on chorioallantoic membrane lesions in experimental fowl Poxvirus infection. Kafkas Üniversitesi Veteriner Fakültesi Dergisi, 15(3), 345-350.

Hitchner, S. B. (2004). History of biological control of poultry diseases in the USA. Avian Diseases, 48(1), 1-8.

Hsu, S. M., Raine, L., \& Fanger, H. X. (1981). Use of avidin-biotin-peroxidase complex $(\mathrm{ABC})$ in immunoperoxidase techniques: A comparison between $\mathrm{ABC}$ and unlabeled antibody (PAP) procedures. Journal of Histochemistry and Cytochemistry, 29(4), 577-580.

Medina, F. M., Ramírez, G. A., \& Hernández, A. (2004). Avian pox in white-tailed laurel-pigeons from the Canary Islands. Journal of Wildlife Diseases, 40(2), 351-355.

Michener, H., \& Michener, J. R. (1936). Abnormalities in Birds. Condor, 38(3), 102-109.

Nuttall, P. A., Brooke, M. D. L., \& Perrins, C. M. (1985). Poxvirus infection of the manx shearwater (Puffinus puffinus). Journal of Wildlife Diseases, 21(2), $120-124$. 
Preis, I. S., Fiúza, A. T. L., Silva, C. C., Braga, J. F. V., Couto, R. M., Martins, N. D. S., \& Ecco, R. (2014). Pathological, immunohistochemical, and molecular findings in commercial laying hens and in backyard chickens naturally infected with the infectious laryngotracheitis virus. Revista Brasileira de Ciência Avícola, 16(4), 359-366.

Pringle, C. R. (1999). Virus taxonomy - 1999. Archives of Virology, 144(2), 421-429.

Reed, W. M., \& Schrader, D. L. (1989). Pathogenicity and immunogenicity of mynah pox virus in chickens and bobwhite quail. Poultry Science, 68(5), 631-638.

Shi, X. M., Zhao, Y., Gao, H. B., Jing, Z., Wang, M., Cui, H. Y., \& Wang, Y. F. (2011). Evaluation of recombinant fowlpox virus expressing infectious bronchitis virus S1 gene and chicken interferon- $\gamma$ gene for immune protection against heterologous strains. Vaccine, 29(8), 1576-1582.

Smits, J. E., Tella, J. L., Carrete, M., Serrano, D., \& López, G. (2005). An epizootic of avian pox in endemic short-toed larks (Calandrella rufescens) and berthelot's pipits (Anthus berthelotti) in the Canary Islands, Spain. Veterinary Pathology Online, 42(1), 59-65.

Suarez, D. L., \& Schultz-Cherry, S. (2000). Immunology of avian influenza virus: A review. Developmental and Comparative Immunology, 24(2), 269-283.
Susta, L., Cornax, I., Diel, D. G., Garcia, S. C., Miller, P. J., Liu, X., \& Afonso, C. L. (2013). Expression of interferon gamma by a highly virulent strain of Newcastle disease virus decreases its pathogenicity in chickens. Microbial Pathogenesis, 61, 73-83.

Tanizaki, E., Kotani, T., Odagiri, Y., \& Horiuchi, T. (1989). Pathologic changes in chickens caused by intravenous inoculation with fowlpox virus. Avian Diseases, 33(2), 333-339.

Tripathy, D. N., Schnitzlein, W. M., Morris, P. J., Janssen, D. L., Zuba, J. K., Massey, G., \& Atkinson, C. T. (2000). Characterization of poxviruses from forest birds in Hawaii. Journal of Wildlife Diseases, 36(2), 225-230.

Weinstein, L., \& Meade, R. H. (1956). Respiratory manifestations of chicken pox: Special consideration of the features of primary varicella pneumonia. AMA Archives of Internal Medicine, 98(1), 91-99.

Winterfield, R. W., \& Reed, W. (1985). Avian pox: Infection and immunity with quail, psittacine, fowl, and pigeon pox viruses. Poultry Science, 64(1), 65-70

Zhao, K., He, W., Xie, S., Song, D., Lu, H., Pan, W., \& Gao, F. (2014). Highly pathogenic fowlpox virus in cutaneously infected chickens, China. Emerging Infectious Diseases, 20(7), 1200. 